EXTENDED REPORT

\title{
Molecular composition and pathology of entheses on the medial and lateral epicondyles of the humerus: a structural basis for epicondylitis
}

\author{
S Milz, T Tischer, A Buettner, M Schieker, M Maier, S Redman, P Emery, D McGonagle, M Benjamin
}

Ann Rheum Dis 2004;63:1015-1021. doi: 10.1136/ard.2003.016378

See end of article for authors' affiliations

\section{Correspondence to:}

Professor M Benjamin, School of Biosciences, Cardiff University, Museum

Avenue, Cardiff CF10

3US, UK; benjamin@ cardiff.ac.uk

Accepted 1 December 2003

\begin{abstract}
Objectives: To improve the understanding of epicondylitis by describing the normal structure and composition of the entheses associated with the medial and lateral epicondyles and their histopathology in elderly cadavers.

Methods: Medial and lateral epicondyles were obtained from 12 cadavers. Six middle aged cadavers (mean 47 years) were used to assess the molecular composition of "normal" entheses from people within an age range vulnerable to epicondylitis. Cryosections of epicondylar entheses were immunolabelled with monoclonal antibodies against molecules associated with fibrocartilage and related tissues. A further six elderly cadavers (mean 84 years) were used for histology to assess features of entheses related to increasing age.

Results: Tendon entheses on both epicondyles fused with those of the collateral ligaments and formed a more extensive structure than hitherto appreciated. Fibrocartilage (which labelled for type II collagen and aggrecan) was a constant feature of all entheses. Entheses from elderly subjects showed extensive microscopic damage, hitherto regarded as a hallmark of epicondylitis.

Conclusions: Fibrocartilage is a normal feature and not always a sign of enthesopathy. Furthermore, pathological changes documented in patients with epicondylitis may also be seen in elderly people. The fusion of the common extensor and flexor tendon entheses with those of the collateral ligaments suggests that the latter may be implicated as well. This may explain why pain and tenderness in epicondylitis may extend locally beyond the tendon enthesis and why some patients are refractory to local treatments.
\end{abstract}

(a) whether fibrocartilage was always a typical feature of epicondylar tendon entheses; $(b)$ the anatomical relationship between the tendon entheses and those of the collateral ligaments, and whether these can be distinguished by their molecular composition; $(c)$ what morphological evidence of degeneration was present in the entheses of elderly people, and how the degenerative changes compare with those described by other authors for epicondylitis.

\section{MATERIALS AND METHODS}

Entheses in middle aged people

Six cadavers of both sexes and of mean age 47 years (range 30-62) were used to assess the tissue type and molecular composition of "normal" entheses typical of people within the age range most commonly associated with epicondylitis. The specimens were obtained from cadavers in the Department of Forensic Medicine (Institut für Rechtsmedizin, Ludwig-Maximilians-Universität) in accordance with the ethical regulations of Munich University. The lateral and medial epicondyles from one limb, together with their associated tendon and collateral ligament entheses, were fixed for 24 hours in $90 \%$ methanol at $4{ }^{\circ} \mathrm{C}$. The tissue was decalcified in 5\% EDTA, rinsed in phosphate buffered saline (PBS), infiltrated for 12 hours with a 5\% sucrose solution in PBS, and sectioned on an HMV500 Microm cryostat at $12 \mu \mathrm{m}$. A radiograph of each specimen was taken before decalcification and the plane of section was as described above.

Abbreviations: $\mathrm{LCL}$, lateral collateral ligament; $\mathrm{MCL}$, medial collateral ligament; PBS, phosphate buffered saline to fulfil our objectives and specifically sought to determine 


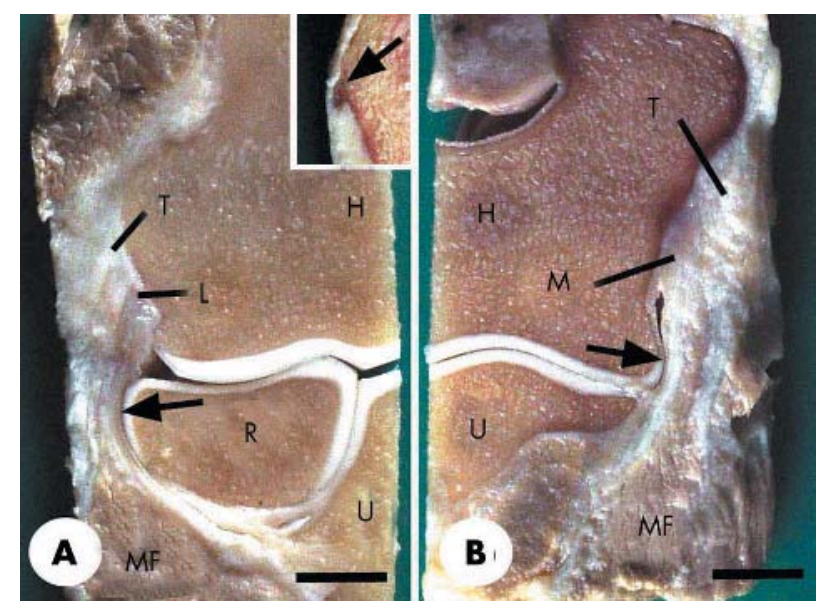

Figure 1 Frontal longitudinal sections of a normal elbow joint from a 30 year old man. (A) The radial (lateral) part of the joint showing the tendinous attachment (T) of the common extensor origin to the lateral epicondyle and the attachment of the lateral collateral ligament (L) with which it was directly continuous. Note that the collateral ligament merges with the annular ligament (arrow), which in turn wraps around the head of the radius (R). Note also the presence of muscle fibres (MF) attaching to both the tendon and the collateral ligament. $\mathrm{H}$, humerus; $\mathrm{U}$, ulna. Inset-the lateral epicondyle from a 47 year old man with a small bony spur (arrow) at the tendon enthesis. (B) The ulnar (medial) part of the same specimen that is featured in (A). The tendinous attachment (T) of the common flexor origin is directly continuous with the medial collateral ligament (M). Note that the ligament wraps around the articular cartilage at the edge of the trochlea (arrow) at the lower end of the humerus (H) and that again there are muscle fibres (MF) attaching to both the tendon and the medial collateral ligament. $U$, ulna; Scale bars $=0.9 \mathrm{~cm}$.

\section{Immunohistochemistry}

Sections from the middle aged subjects were immunolabelled with a panel of primary monoclonal antibodies directed against collagens (types I, II, III, and VI), glycosaminoglycans (chondroitin-4- and -6-sulphates, keratan and dermatan sulphates), and proteoglycans (aggrecan, link protein, versican, and tenascin). Details of the primary antibodies (sources, dilutions, and enzyme pretreatments) have been given previously. ${ }^{16}$ Endogenous peroxidase activity was blocked by pretreating sections with $0.3 \%$ hydrogen peroxide in methanol, and non-specific binding of the secondary antibody (horse antimouse; provided in the Vectastain ABC "Elite" avidin/biotin/peroxidase detection kit) was reduced by treating the sections with horse serum. Control sections were made by omitting the primary antibody.

\section{Entheses in elderly people}

To assess the pathological features of epicondylar entheses associated with elderly people, the lateral and/or medial epicondyles together with their associated entheses were removed from six dissecting room cadavers (donated to Cardiff University) of both sexes (mean age 84 years; range 78-101) that had been perfused with a solution containing formaldehyde and alcohol. Details of this have been given previously. ${ }^{17}$ Although none of the cadavers selected showed any apparent gross abnormality in the elbow region, the use of elderly dissecting room specimens increased the likelihood of finding degenerative changes at the entheses. All specimens were postfixed for 1 week in $10 \%$ neutral buffered formal saline, decalcified in 5\% nitric acid, dehydrated with graded alcohols, cleared in xylene, and embedded in paraffin wax. Radiographs were taken before decalcification. After histological processing, longitudinal sections of each epicondylar enthesis were cut in the coronal (frontal) plane at $8 \mu \mathrm{m}$ and stained with Masson's trichrome. The chosen plane of

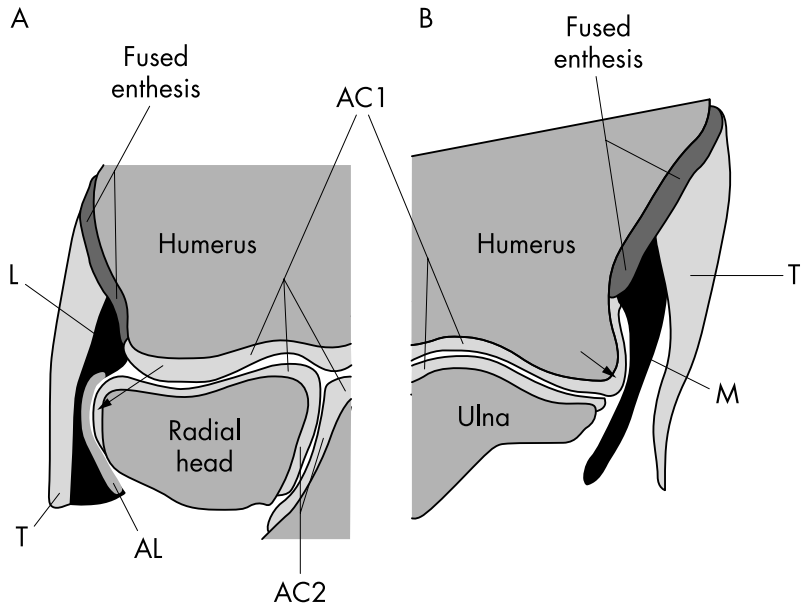

Figure 2 A schematic representation of the enthesis organs associated with the medial and lateral epicondyles based on fig 1. The elbow is thus drawn in coronal (frontal) section with the humerus located proximally and the radius and ulna distally. Both the articular cartilages of the elbow $(\mathrm{AC} 1)$ and superior radioulnar (AC2) joints are visible. (A) The lateral epicondyle. The lateral collateral ligament (L) fuses with the annular ligament (AL), which in turn wraps around the radial head at the position of the arrow. The common extensor tendon (T) lies immediately lateral to the collateral ligament and the entheses of these two structures are fused. The single fused enthesis serves to dissipate stress concentration between tendon and ligament at the bony interface and stress is further reduced by the contact between annular ligament and radial head (arrow). Consequently, the enthesis organ comprises the tendon, collateral ligament, annular ligament, and the adjacent circumference of the radial head. (B) The medial epicondyle. The medial collateral ligament (M) fuses with the tendon (T) of the common flexor origin at its enthesis and the former presses against the articular cartilage on the edge of the humerus at the position of the arrow. The enthesis organ thus consists of the tendon, collateral ligament, and a part of the humeral articular cartilage.

section ensured that both the tendon of the common extensor or flexor origins and the associated lateral/medial collateral ligaments of the elbow were present in the slides examined (fig 1).

\section{RESULTS}

The tendon attachments to both epicondyles were directly continuous with those of the collateral ligaments as indicated in the gross section of the normal elbow of a 30 year old man illustrated in fig 1 . Figure 2 shows a schematic representation of this section. In all specimens the epicondylar attachment sites of both the flexor and extensor muscles of the forearm blended imperceptibly with the humeral attachments of the medial (MCL) and lateral collateral (LCL) ligaments (fig lA). Thus, as a result of the fusion between ligament and tendon at the attachments to each epicondyle, there was a single enthesis with no boundary between the two structures which formed it. Elsewhere, each tendon and its adjacent ligament could be more easily distinguished. As both ligaments also pressed against articular cartilage, and the LCL was attached to the annular ligament (figs 1A, 2, 3A), the fused tendonligament attachment sites on either side of the elbow, each formed part of an "enthesis organ" that dissipated stress concentration over a broad area.

In three of the six specimens from middle aged subjects, a small bony spur (enthesophyte) was present at the proximal part of the attachment of the common extensor origin (fig 1A inset). These spurs were also visible in the accompanying radiographs (not illustrated). However, no spurs were found in any of the specimens from the medial side of the elbow. In all specimens, both the medial and lateral epicondylar 


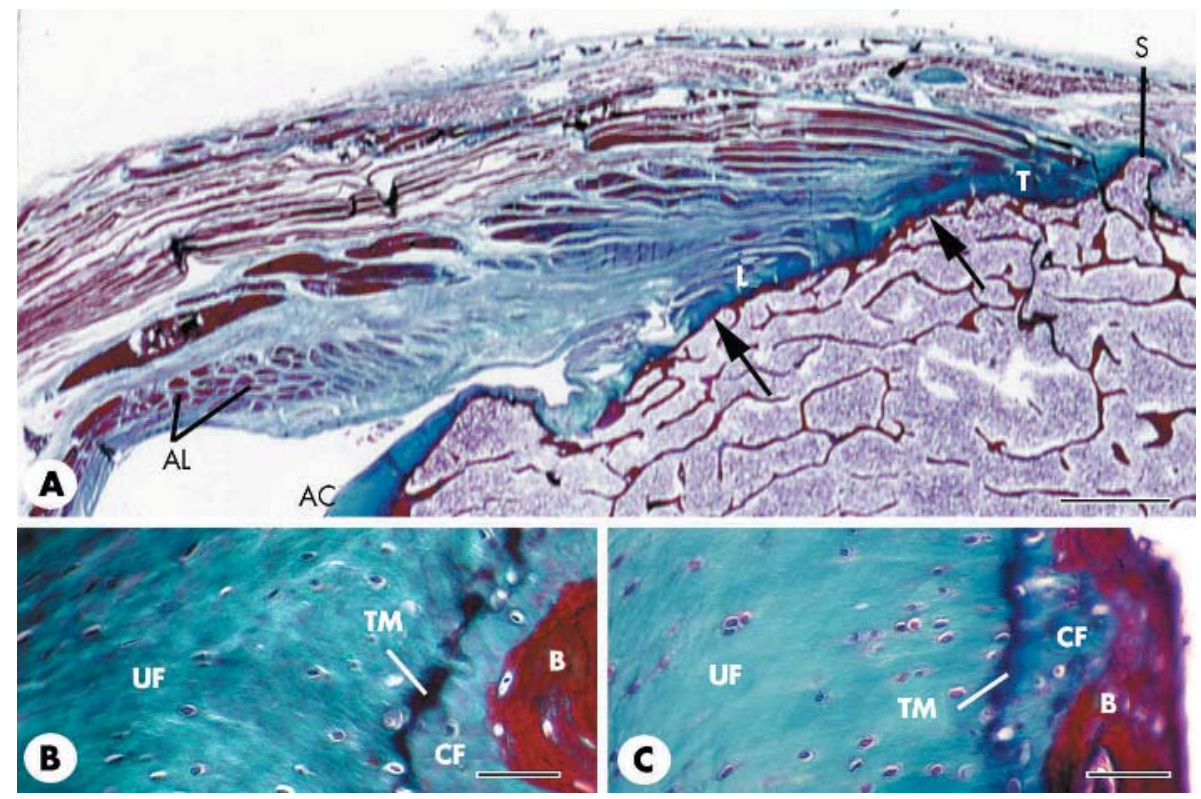

Figure 3 Routine histology and histopathology of the medial and lateral epicondylar entheses. (A) A low power view (frontal section) of the single enthesis on the lateral epicondyle from a 101 year old woman that is formed by the fusion of the tendon (T) of the common extensor origin with the lateral collateral ligament (L) of the elbow joint. The tendon and collateral ligament similarly fuse with each other at the medial epicondylar enthesis (not illustrated). Note that the lateral collateral ligament is also fused with the annular ligament (AL) that wraps around around the head of the radius (not visible in the section). Note also the presence of only a thin layer of subchondral bone (arrows) throughout the enthesis and the small bony spur (S) at the proximal end of the tendon attachment. AC, articular cartilage. Scale bar $=0.2 \mathrm{~cm}$. (B, C) Fibrocartilage at the attachment of both the tendon (B) and the ligament $(C)$ to the lateral epicondyle in the same specimen featured in (A). The fibrocartilage is equally prominent at both sites and consists of zones of uncalcified (UF) and calcified (CF) tissue separated by a calcification front known as the tidemark (TM). B, bone. Scale bars $=50 \mu \mathrm{m}$.

entheses were moderately fibrocartilaginous, and zones of uncalcified and calcified fibrocartilage separated by one or more tidemarks were clearly visible in both middle aged and elderly subjects (figs 3B, and C). The underlying layer of subchondral bone was thin (generally $<150 \mu \mathrm{m}$ ) and thus most of the bone at the entheses was cancellous (fig 3A).

\section{Molecular composition of the entheses}

Table 1 summarises the molecular composition of the entheses and features of special interest are described below and illustrated in Figs 4A-S. In both the tendon and ligament parts of the lateral epicondylar enthesis, the uncalcified and calcified fibrocartilage zones immunolabelled for type II

Table 1 Summary of the immunohistochemical labelling characteristics of the entheses associated with the lateral and medial epicondyles

\begin{tabular}{|c|c|c|c|c|c|c|c|c|c|c|c|c|}
\hline & \multicolumn{4}{|c|}{ Collagens } & \multicolumn{4}{|c|}{ Glycosaminoglycans } & \multicolumn{4}{|c|}{ Proteoglycans and glycoproteins } \\
\hline & I & II & IIII & VI & KS & DS, C4S & C4S & C6S & Agg & Link & Versican & Tenascin \\
\hline \multirow{2}{*}{\multicolumn{13}{|c|}{$\begin{array}{l}\text { Lateral epicondyle } \\
\text { Tendon attachment }\end{array}$}} \\
\hline & & & & & & & & & & & & \\
\hline Bone matrix & 6 & 0 & 0 & 6 & 0 & 0 & 0 & 0 & 0 & 0 & 0 & 0 \\
\hline Fibrocartilage & $6^{*}$ & 6 & 6 & 6 & 6 & 6 & 6 & 6 & 6 & 6 & $6^{*}$ & $6^{*}$ \\
\hline Fibrous zone & 6 & 3 & 6 & 6 & 6 & 6 & 6 & 4 & 3 & 3 & 6 & 4 \\
\hline \multicolumn{13}{|c|}{ Ligament attachment } \\
\hline Bone matrix & 6 & 0 & 0 & 6 & 0 & 0 & 0 & 0 & 0 & 0 & 0 & 0 \\
\hline Fibrocartilage & $6^{*}$ & 6 & 6 & 6 & 6 & 6 & 6 & 6 & 6 & 6 & $6^{*}$ & $6^{*}$ \\
\hline Fibrous zone & 6 & 3 & 6 & 6 & 6 & 6 & 6 & 4 & 3 & 3 & 6 & 4 \\
\hline \multicolumn{13}{|c|}{ Medial epicondyle } \\
\hline Bone matrix & 6 & 0 & 0 & 6 & 0 & 0 & 0 & 0 & 0 & 0 & 0 & 0 \\
\hline Fibrocartilage & 6 & 6 & 6 & 6 & 6 & 6 & 6 & 6 & 6 & 6 & 6 & 6 \\
\hline Fibrous zone & 6 & 3 & 6 & 6 & 6 & 6 & 6 & 3 & 2 & 3 & 6 & 4 \\
\hline \multicolumn{13}{|c|}{ Ligament attachment } \\
\hline Bone matrix & 6 & 0 & 0 & 6 & 0 & 0 & 0 & 0 & 0 & 0 & 0 & 0 \\
\hline Fibrocartilage & 6 & 6 & 6 & 6 & 6 & 6 & 6 & 6 & 6 & 6 & 6 & 6 \\
\hline Fibrous zone & 6 & 3 & 6 & 6 & 6 & 6 & 6 & 1 & 2 & 3 & 6 & 4 \\
\hline
\end{tabular}



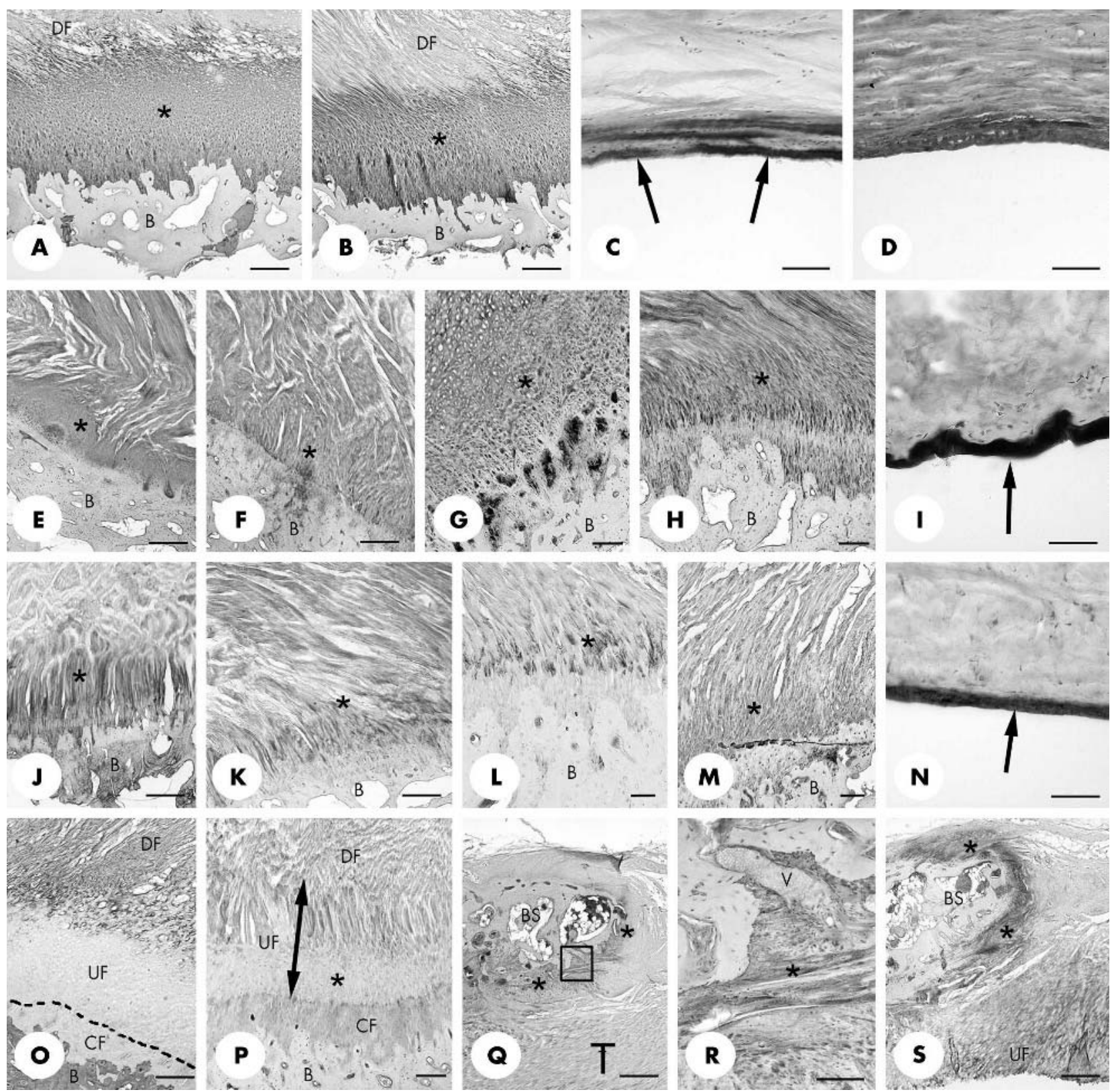

Figure 4 Immunohistochemical labelling of the medial and lateral epicondylar entheses. (A, B) Extensive labelling of fibrocartilage for type II collagen (asterisk) at both the tendon (A) and ligament (B) parts of the lateral epicondylar enthesis. B, bone; DF dense fibrous connective tissue. Scale bars $=500 \mu \mathrm{m}$. (C) Strong labelling for type II collagen (arrows) in the region of the medial collateral ligament close to the enthesis, where it wraps around the edge of the humeral articular cartilage. The region illustrated is that indicated by the arrow in fig 1B. Note that only the surface of the ligament is labelled. Scale bar $=100 \mu \mathrm{m}$. (D) At the lateral epicondylar enthesis, labelling of the "wrap-around" region of the lateral collateral ligament (that is, where it was in contact with the head of the radius - see arrow in fig 1A) for type II collagen was seen not only at the ligament surface but also in the underlying tissue. Scale bar $=100 \mu \mathrm{m}$. (E, F) Aggrecan labelling (asterisk) of fibrocartilage at the ligament (E) and tendon (F) parts of the medial epicondylar enthesis. B, bone. Scale bars $=500 \mu \mathrm{m}$. (G, H) Aggrecan labelling (asterisk) of fibrocartilage at the ligament (G) and tendon (H) parts of the lateral epicondylar enthesis. B, bone. Scale bars $=250 \mu \mathrm{m}$. (I) Intense labelling for aggrecan at the surface (arrow) of the "wrap-around" region of the lateral collateral ligament (that is, where it was in contact with the head of the radius - see arrow in fig $1 \mathrm{~A})$. Scale bar $=50 \mu \mathrm{m}$. (J, K) Labelling for link protein (asterisk) in fibrocartilage at both the ligament $(\mathrm{J})$ and tendon $(\mathrm{K})$ parts of the lateral epicondylar enthesis. B, bone. Scale bars $=500 \mu \mathrm{m}$. (L) Chondroitin-6-sulphate labelling (asterisk) in fibrocartilage in the tendon part of the medial epicondylar enthesis. B, bone. Scale bar $=100 \mu \mathrm{m}$. (M) Chondroitin-6-sulphate labelling (asterisk) in fibrocartilage in the ligament part of the lateral epicondylar enthesis. B, bone. Scale bar $=100 \mu \mathrm{m}$. (N) Strong labelling for chondroitin-6-sulphate (arrow) in the region of the lateral collateral ligament close to the enthesis, where it wraps around the head of the radius. The region illustrated is that indicated by the arrow in fig 1 A. Scale bar $=50 \mu \mathrm{m}$. (O) Type I collagen labelling in the ligament part of the lateral epicondylar enthesis. Note the strong labelling of both the dense fibrous zone of the ligament enthesis (DF) and the bone (B), but the absence of labelling from the calcified (CF) and much of the uncalcified enthesis fibrocartilage (UF). The dotted line indicates the position of the tidemark. Scale bar $=500 \mu \mathrm{m}$. (P) Versican labelling in the ligament part of the lateral epicondylar enthesis. Labelling is present in the dense fibrous zone (DF), part of the adjacent zone of uncalcified fibrocartilage (UF), and the zone of calcified fibrocartilage (CF). However, labelling was largely absent from the deeper part of the zone of uncalcified fibrocartilage (asterisk). B, bone. Scale bar $=250 \mu \mathrm{m}$. (Q) Low power view of type II collagen labelling (asterisk) of fibrocartilage at the surface of a small bony spur (BS) in the tendon (T) part of the lateral epicondylar enthesis. Scale bar $=500 \mu \mathrm{m}$. (R) High power view of the region enclosed in the rectangle in (Q) showing a small venule (V) packed with blood cells, passing between tendon and bone in the type II collagen positive region (asterisk). Scale bar $=250 \mu \mathrm{m}$. (S) Strong labelling for link protein (asterisk) around the tip of the bony spur (BS) featured in (Q) and (R) and in the uncalcified fibrocartilage (UF) of the tendon. A similar labelling pattern was also evident for aggrecan. Scale bar $=500 \mu \mathrm{m}$. 


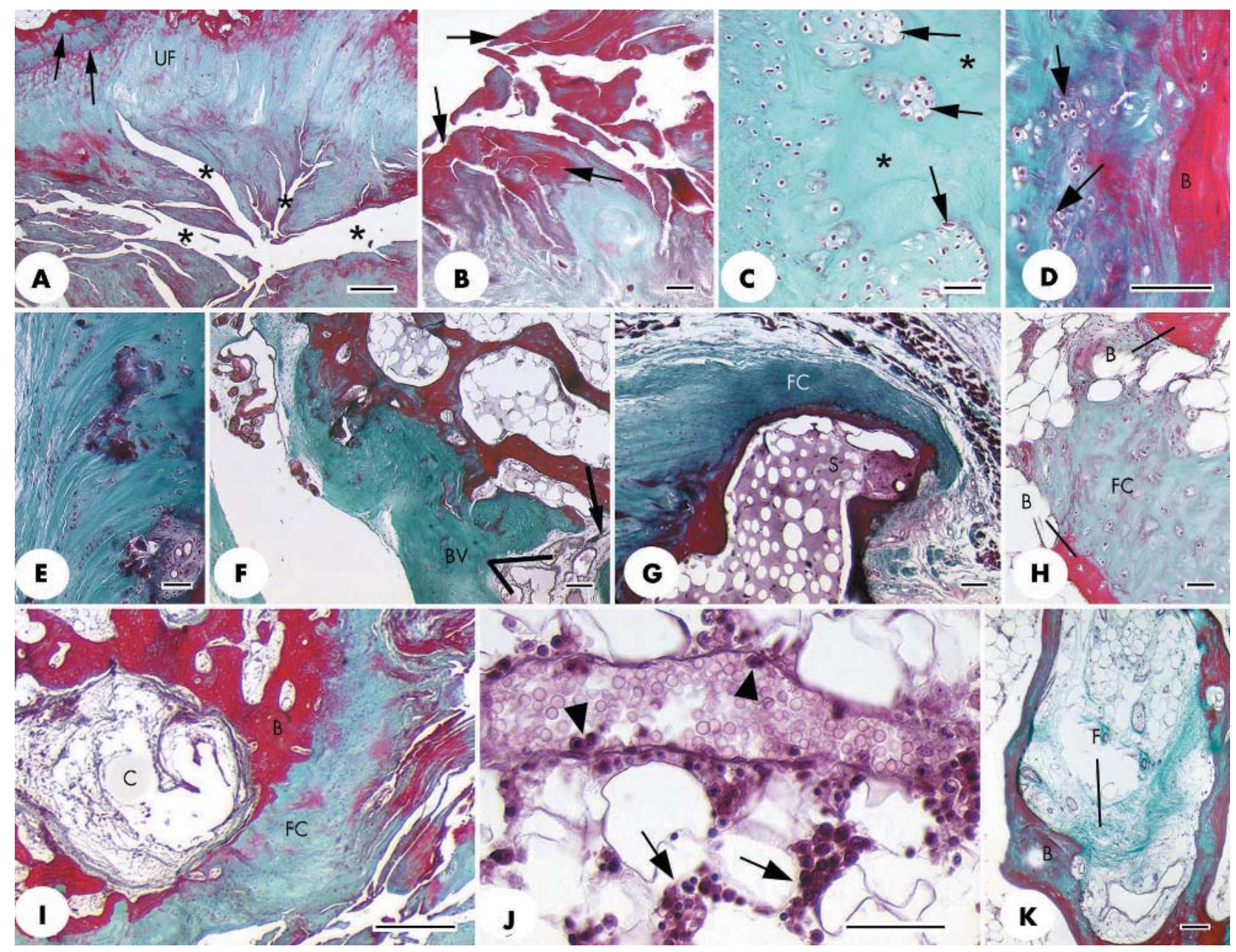

Figure 5 (A) Prominent fissures (*) at the ligament end of the medial epicondylar enthesis that greatly disrupt the normal structure of the attachment site. UF, uncalcified fibrocartilage; arrows, tidemarks. Scale bar $=500 \mu \mathrm{m}$. (B) Higher power view of several fissures from the same specimen as illustrated in (A) to show the prominent layer of fibrin (arrows) on the surface of the fissures. Scale bar $=50 \mu \mathrm{m}$. (C) Clusters of fibrocartilage cells (arrows) separated by small regions of acellular matrix (asterisk) in the tendon part of a lateral epicondylar enthesis. Scale bar $=50 \mu \mathrm{m}$. (D) Fibrocartilage cell proliferation (arrows) at the tendon attachment on the lateral epicondylar enthesis. B, bone. Scale bar = $100 \mu \mathrm{m}$. (E) Small foci of calcified tissue and fibrocartilage cell clusters in the zone of uncalcified fibrocartilage at the medial epicondylar enthesis. Scale bar $=50 \mu \mathrm{m}$. (F) Numerous small blood vessels (BV), indicating vascular proliferation at the site of a small bony defect (arrow) in the tendon attachment on the medial epicondylar enthesis. The blood vessels are passing between the tendon and the bone marrow. Scale bar $=100 \mu \mathrm{m}$. (G) A section adjacent to that featured in fig $2 A$ to show the presence of fibrocartilage (FC) at the tip of the small bony spur (S). (H) A small region of fibrocartilage (FC) within the bone marrow, between neighbouring bone spicules (B). Scale bar $=50 \mu \mathrm{m}$. (I) A subchondral bone cyst (C) beneath the tendinous part of the lateral epicondylar enthesis. B, bone; FC, fibrocartilage. Scale bar $=500 \mu \mathrm{m}$. (J) A venule in the bone marrow associated with the tendinous part of the lateral epicondylar enthesis. Note the presence of inflammatory cells around the vessel (arrows) and of several marginating leucocytes around the edge of the lumen (arrowheads). Scale bar $=50 \mu \mathrm{m}$. (K) Evidence of bone marrow fibrosis (F) in the same specimen as (J). B, bone. Scale bar $=100 \mu \mathrm{m}$. All sections were stained with Masson's trichrome.

collagen, chondroitin-6-sulphate, aggrecan, and link protein (figs $4 \mathrm{~A}, \mathrm{~B}, \mathrm{G}, \mathrm{H}$ ). In three cadavers, aggrecan and link protein labelling extended into the fibrous zones of both the ligament and tendon, away from the insertion site. This was especially evident in the region where the LCL passed over the edge of the radial head (fig 4D). Although type I collagen, tenascin, and versican labelling were widespread in the midsubstance of the LCL, labelling for these molecules was sometimes absent in its fibrocartilage zones. The bony spurs seen in three cadavers were located in the most proximal parts of the lateral epicondylar enthesis-that is, in association with the tendon part of the attachment site. They were surrounded by fibrocartilage that labelled as described above.

In general, the molecular composition of the medial epicondylar enthesis was similar to that described for the lateral epicondyle. However, labelling for typical fibrocartilage molecules (type II collagen, chondroitin-6-sulphate, aggrecan, and link protein) was less extensive than in the lateral epicondylar enthesis (figs $4 \mathrm{E}$ and $\mathrm{F}$ ). Furthermore, there was local absence of type I collagen, tenascin, and versican labelling on the lateral but not the medial epicondylar enthesis, and no bony spurs were seen in any specimens at the latter location. The MCL showed some evidence of fibrocartilage differentiation where the ligament passed over the edge of the humeral articular cartilage (fig 4C). Here, the tissue labelled for type II collagen, aggrecan, and link protein.

\section{Enthesis histopathology}

Signs of degenerative change were common in both the ligament and tendon entheses on either side of the elbow. The organised enthesis structure was most strikingly disrupted by the presence of numerous fissures (fig 5A). The edges of these were sometimes lined by a layer of brightly 
staining fibrin (figs 5B). Chondrocytic clusters and signs of fibrocartilage cell proliferation were also a striking feature of some of the material from the elderly subjects (figs 5C and D) and there were small foci of intratendinous calcification (fig 5E). Areas where several small blood vessels were aggregated together near the bony interface suggested vascular proliferation and the potential for some vessels in the tendon/ligament to communicate with those in the bone marrow (fig 5F).

The tips of the bony spurs seen at the lateral epicondyle in two of the six specimens, were covered with fibrocartilage (fig 5G) and in one of these, islands of fibrocartilage were present within the bone itself, between adjacent bone spicules (fig $5 \mathrm{H}$ ). In the same cadaver, there was a small bone cyst (fig 5I). Although evidence of inflammation was rare, inflammatory cells were occasionally present around small venules in the bone marrow (fig 5J). This was accompanied by some evidence of bone marrow fibrosis (fig $5 \mathrm{~K}$ ), possibly indicating previous inflammation at those sites.

\section{DISCUSSION}

The purpose of this study was to describe the normal structure and molecular composition of entheses implicated in epicondylitis, and to highlight the presence of degenerative changes in elderly subjects that are similar to those described by others in patients with tennis or golfer's elbow. As in all studies of this type, there might have been undocumented episodes of epicondylitis earlier in the subjects' lives, despite our efforts to select "normal" elbows'. However, this is unlikely to be a significant issue, as the general incidence of epicondylitis is less than $1 \% .^{18}$

We showed that $(a)$ tendon entheses on both the medial and lateral sides of the elbow formed complex structures that were totally integrated with the attachments of the respective collateral ligaments. Thus, abnormality in the tendon or ligament is likely to affect the other, and both may be involved in epicondylitis. This may be why some cases of epicondylitis are refractory to tenodesis ${ }^{19-22} ;(b)$ fibrocartilage is a constant feature of all entheses from subjects of a wide age range and its mere presence cannot be considered as "pathological" itself; (c) entheses from elderly people can show extensive microscopic evidence of degeneration, hitherto equated specifically with epicondylitis.

From a clinical perspective, epicondylitis is sometimes associated with pain and tenderness adjacent to the tendon attachments, ${ }^{5}$ and degenerative changes have been reported in the collateral ligament by magnetic resonance imaging and sonography. ${ }^{4}$ We think the involvement of structures other than the tendon enthesis, partly reflects the complex nature of the attachment sites that we have described. On both epicondyles the tendon and ligament attachments merged imperceptibly with each other and had a similar labelling profile for molecules found in numerous other fibrocartilaginous entheses. ${ }^{14}{ }^{16} 23-27$ This is the first demonstration of fusion between the tendons and the collateral ligaments at their entheses, but a close association has been noted previously by surgical anatomists and radiologists. ${ }^{4}$ Many of the older gross anatomy texts, in particular, comment on such matters. Thus, both Strasser ${ }^{28}$ and Robinson ${ }^{29}$ pointed out that the collateral ligaments are intimately associated with the muscles which originate from the medial and lateral epicondyles, and Rüdinger ${ }^{30}$ noted that the extensor carpi radialis brevis is attached to the joint capsule and can put tension upon it. We contend that on both sides of the elbow, the conjoined tendon-ligament enthesis created a wide insertional area that can be viewed as part of an "enthesis organ" that dissipates stress concentration away from a small focal point. ${ }^{14}$
We view enthesis fibrocartilage as a sign of normal functional adaptation of the epicondylar attachment sites that reduces the risk of wear and tear. ${ }^{11-15}$ As at entheses elsewhere in the body, ${ }^{11}{ }^{12}$ fibrocartilage promotes stress dissipation at the hard-soft tissue interface by enabling them to withstand compression and/or shear forces. This is reflected by its molecular composition-that is, the presence of molecules typical of hyaline cartilage (aggrecan, link protein, and type II collagen-see the review by Benjamin and McGonagle $^{14}$ ), and the local absence of those typical of fibrous tissue (type I collagen and versi$\left.\operatorname{can}^{23}{ }^{31}\right)$. The normal presence of fibrocartilage in the epicondylar entheses is in line with the report of Putz et al, ${ }^{31}$ who commented on it at the collateral ligament entheses in their general account of the functional anatomy of the elbow joint.

Histopathological changes similar to those described in reports on epicondylitis-notably, fibrovascular proliferation, ${ }^{10} 3233$ focal calcifications, ${ }^{34}$ and "mucoid degeneration" or fibrocartilage proliferation, ${ }^{4}$ were seen in both the ligamentous and tendinous parts of the medial and lateral epicondylar entheses. As in epicondylitis, the majority of changes seen in elderly cadavers were degenerative rather than inflammatory. The widespread nature of such changes suggests that degeneration is normal in elderly people. This is in line with the earlier findings of Kumagai et al on the rotator cuff entheses of older subjects. ${ }^{35}$ Our findings also suggest that there are no histopathological changes that can be regarded as specific to epicondylitis. All the major changes described by other authors in patients with epicondylitis (see above) have also been seen by us in the entheses of elderly subjects. We suggest that fibrocartilage proliferation in epicondylitis reflects the importance of mechanical forces in understanding the causative factors of these conditions. ${ }^{236}$ Mechanical overload may also induce bony spur formation in lateral epicondylar enthesis fibrocartilage-as in the dorsal capsule of the lumbar facet joints. ${ }^{24}$ However, it must be accepted that some epidemiological data on epicondylitis are conflicting and do not always suggest a close link with strenuous manual work. ${ }^{18}$

We have shown that aggrecan and link protein are present in all enthesis fibrocartilages examined, as in several other regions of the body (see the review by Benjamin and McGonagle $^{14}$ ). As cleavage of aggrecan by matrix metalloproteinases is a feature of cartilage degeneration, ${ }^{37}$ this probably contributes to "mucoid (mucinoid) degeneration" at epicondylar entheses. ${ }^{2}$ Because link protein is known to be an antigenic target for autoimmune responses in rheumatoid arthritis, ${ }^{38-40}$ it is possible that some cases of epicondylitis may also be related to this condition. It is pertinent to note that Lehtinen et al have shown that the lateral epicondyle is affected by erosion in $78 \%$ of patients with a 15 year history of seropositive and erosive rheumatoid arthritis. ${ }^{41}$ Finally, although lateral epicondylitis is usually regarded as idiopathic, it is worth noting that the lateral epicondylar enthesis has been listed among the most commonly affected in patients with spondyloarthropathy. ${ }^{42}$ This may also have a bearing on autoimmune responses to fibrocartilage antigens. ${ }^{43}$

In summary, we conclude that fibrocartilage is a normal feature of epicondylar entheses that should not merely be equated with pathological change. Furthermore, none of the degenerative changes previously associated with epicondylitis are specific to those diseases, but can also be a sign of aging. Finally, clinical observations of diffuse pain and tenderness in epicondylitis (that is, that which extends locally beyond the actual tendon enthesis to bone) may relate to the complex nature of the attachment site and the presence of an enthesis organ. 


\section{ACKNOWLEDGEMENTS}

This work was supported by Action Medical Research and Search. Professor McGonagle is an MRC clinical scientist and Professor Emery is an ARC professor of rheumatology.

\section{Authors' affiliations}

S Milz, T Tischer, Anatomische Anstalt, Ludwig-Maximilians-Universität, Munich, Germany

A Buettner, Institut für Rechtsmedizin, Ludwig-Maximilians-Universität, Munich, Germany

M Schieker, Chirurgische Klinik und Poliklinik, Ludwig-Maximilians-

Universität, Munich, Germany

M Maier, Orthopädische Klinik und Poliklinik, Ludwig-Maximilians-

Universität, Munich, Germany

S Redman, M Benjamin, Cardiff School of Biosciences, University of Cardiff, UK

P Emery, D McGonagle, Department of Rheumatology, Leeds Hospital Medical School, UK

\section{REFERENCES}

1 Nirschl RP. Elbow tendinosis/tennis elbow. Clin Sports Med 1992;11:851-70

2 Field LD, Savoie FH. Common elbow injuries in sport. Sports Med 1998;26:193-205.

3 Williams PL, Warwick R. Gray's anatomy. Edinburgh: Churchill Livingstone, 1980.

4 Potter HG, Hannafin JA, Morwessel RM, DiCarlo EF, O'Brien SJ, Altchek DW. Lateral epicondylitis: correlation of MR imaging, surgical and histopathologic findings. Radiology 1995;196:43-6.

5 Chard MD. The elbow. In: Klippel JH, Dieppe PA, eds. Rheumatology. 2nd ed. London: Mosby, 1998:4 8.1-9.8

6 Connell D, Burke F, Coombes P, McNealy S, Freeman D, Pryde D, et al. Sonographic examination of lateral epicondylitis. AJR Am J Roentgenol 2001; 176:777-82.

7 Yahia H, Drouin G, Maurais G, Garzon S, Rivard CH. Degeneration of the human lumbar spine ligaments. An ultrastructural study. Pathol Res Pract 1989; 184:369-75.

8 Putz R, Reichelt A. Strukturelle Befunde am Lig. coracoacromiale bei Rotatorenmanschettenruptur, Tendinosis calcarea und Supraspinatussyndrom. Z Orthop Ihre Grenzgeb 1990;128:46-50.

9 Chard MD, Cawston TE, Riley GP, Gresham GA, Hazleman BL. Rotator cuff degeneration and lateral epicondylitis: a comparative histological study. Ann Rheum Dis 1994;53:30-4.

10 Galliani I, Burattini S, Mariani AR, Riccio M, Cassiani G, Falcieri E. Morpho-functional changes in human tendon tissue. Eur J Histochem 2002;46:3-12.

11 Schneider H. Zur Struktur der Sehnenansatzzonen. Z Anat 1956;119:431-56.

12 Benjamin M, Evans EJ, Copp L. The histology of tendon attachments in man. $J$ Anat 1986;149:89-100.

13 Woo SL-Y, Maynard J, Butler D, Lyon R, Torzilli P, Akeson W, et al. Ligament tendon, and joint capsule insertions to bone. In: Woo SL-Y, Buckwalter JA, eds. Injury and repair of the musculoskeletal soft tissues. Illinois: Amer Acad Orthop Surg, 1988:133-66.

14 Benjamin M, McGonagle D. The anatomical basis for disease localisation in seronegative spondyloarthropathy at entheses and related sites. J Anat 2001;199:503-26.

15 Benjamin M, Kumai T, Milz S, Boszczyk BM, Boszczyk AA, Ralphs JR. The skeletal attachment of tendons - tendon entheses. Comp Biochem Phys A Mol Integr Physiol 2002;133:931-45.

16 Milz S, Valassis G, Büttner A, Maier M, Putz R, Ralphs JR, et al. Fibrocartilage in the transverse ligament of the human acetabulum. I Anat $2000 ; 198: 223-8$.
17 Rufai A, Ralphs JR, Benjamin M. Structure and histopathology of the insertional region of the human Achilles tendon. J Orthop Res 1995;13:585-93.

18 Viikari-Juntura $E$. The role of physical stresses in the development of hand/ wrist and elbow disorders. In: Gordon SL, Blair SJ, Fine L, eds. Repetitive motion disorders of the upper extremity. Illinois: Amer Acad Orthop Surg, 1995:7-30.

19 Grundberg AB, Dobson JF. Percutaneous release of the common extensor origin for tennis elbow. Clin Orthop 2000;376:137-40.

20 Bennett JB. Lateral and medial epicondylitis. Hand Clin 1994;10:157-63.

21 Plancher KD, Halbrecht J, Lourie GM. Medial and lateral epicondylitis in the athlete. Clin Sports Med 1996;15:283-305.

22 Das D, Maffulli N. Surgical management of tennis elbow. J Sports Med Phys Fitness 2002:42:190-7.

23 Boszczyk AA, Boszczyk BM, Putz R, Benjamin M, Milz S. Expression of a wide range of fibrocartilage molecules at the entheses of the alar ligamentspossible antigenic targets for rheumatoid arthritis? I Rheumatol 2003;30:1420-5.

24 Boszczyk BM, Boszczyk AA, Korge A, Grillhosl A, Boos WD, Putz R, et al. Immunohistochemical analysis of the extracellular matrix in the posterior capsule of the zygapophysial joints in patients with degenerative L4-5 motion segment instability. J Neurosurg 2003;99(suppl 1):27-33.

25 Boszczyk BM, Boszczyk AA, Putz R, Büttner A, Benjamin M, Milz S. An immunohistochemical study of the dorsal capsule of the lumbar and thoracic facet joints. Spine $2001 ; 26$ : E338-43.

26 Milz S, Schlüter T, Putz R, Moriggl B, Ralphs JR, Benjamin M. Fibrocartilage in the transverse ligament of the human atlas. Spine 2001;26:1765-71.

27 Moriggl B, Jax P, Milz S, Büttner A, Benjamin M. Fibrocartilage at the entheses of the suprascapular (superior transverse scapular) ligament of mana ligament spanning two regions of a single bone. J Anat 2001;199:539-45.

28 Strasser H. Lehrbuch der Muskel- und Gelenkmechanik. IV Band: Spezieller Teil, Die obere Extremität. Berlin: Springer, 1917.

29 Robinson A. Cunningham's textbook of anatomy. 4th ed. Edinburgh: Frowde, Hodder \& Stoughton, 1913.

30 Rüdinger N. Topographisch-chirurgische Anatomie des Menschen. Stuttgart: J.G. Cotta, 1874.

31 Putz R, Milz S, Maier M, Boszczyk A. Funktionelle Morphologie des Ellenbogengelenks. Orthopäde 2003;32:684-90.

32 Regan W, Wold LE, Coonrad R, Morrey BF. Microscopic histopathology of chronic refractory lateral epicondylitis. Am $j$ Sports Med 1992;20:746-9.

33 Steinborn M, Heuck A, Jessel C, Bonel H, Reiser M. Magnetic resonance imaging of lateral epicondylitis of the elbow with a $0.2-\mathrm{T}$ dedicated system. Eur Radiol 1999;9:1376-80

34 Galliani I, Columbaro M, Ferri S, Valmori A, Cassiani G, Maltarello MC, et al. Calcific chronic lateral epicondylitis: a histological and ultrastructural study. J Submicrosc Cytol Pathol 1997;29:453-9.

35 Kumagai J, Sarkar S, Uhthoff HK. The collagen types in the attachment zone of rotator cuff tendons in the elderly: an immunohistochemical study. J Rheumatol 1994:2096-100.

36 Kandemir U, Fu FH, McMahon PJ. Elbow injuries. Curr Opin Rheumatol 2002;14:160-7.

37 Caterson B, Flannery CR, Hughes CE, Little CB. Mechanisms involved in cartilage proteoglycan catabolism. Matrix Biol 2000;19:333-44.

38 Guerassimov A, Duffy C, Zhang Y, Baneriee S, Leroux JY, Reimann A, et al. Immunity to cartilage link protein in patients with juvenile rheumatoid arthritis. $J$ Rheumatol 1997:24:959-64.

39 Guerassimov A, Zhang Y, Baneriee S, Cartman A, Webber C, Esdaile J, et al. Autoimmunity to cartilage link protein in patients with rheumatoid arthritis and ankylosing spondylitis. J Rheumatol 1998;25:1480-4.

40 Zhang Y, Guerassimov A, Leroux JY, Cartman A, Webber C, Lalic R, et al. Induction of arthritis in $\mathrm{BALB} / \mathrm{c}$ mice by cartilage link protein: involvement of distinct regions recognized by T and B lymphocytes. Am J Pathol 1998; 153:1283-91.

41 Lehtinen JT, Kaarela K, lkavalko M, Kauppi MJ, Belt EA, Kuusela PP, et al. Incidence of elbow involvement in rheumatoid arthritis. A 15 year endpoint study. J Rheumatol 2001;28:70-4.

42 Braun J, Khan MA, Sieper J. Enthesitis and ankylosis in spondyloarthropathy: what is the target of the immune response? Ann Rheum Dis 2000;59:985-94.

43 Maksymowych WP. Ankylosing spondylitis-at the interface of bone and cartilage. J Rheumatol 2000;27:2295-301. 